\section{International Scientific Journal Theoretical \& Applied Science}

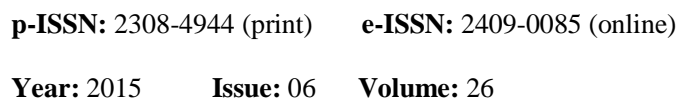

SECTION 31. Economic research, finance, innovation, risk management.
Anatoly Aleksandrovich Naumov Docent, Candidate of Technical Sciences, Center of Applied Mathematical Research, Novosibirsk, Russia a_a_naumov@mail.ru

Anastasia Anatolievna Naumova Marketing Director, MBA, Marie Forleo Business School, NYC, Manchester, UK anastasia.naumova@yahoo.com

\title{
TO AN ESTIMATION OF EFFICIENCY OF INNOVATIVE PROJECTS ON THE BASIS OF DETAIL OF FINANCIAL FLOWS
}

Abstract: The paper discusses the problems of some methods of analysis of investment and innovative projects on the basis of a given income. The proposed scheme of evaluation of project effectiveness with regard to integration on the basis of detail of financial flows. The methods of estimating the rate of return and payback period. Proposed general approaches and algorithms to the evaluation of the effectiveness of integrated projects.

Key words: Investment projects, innovative projects, efficiency, efficiency criteria, NFV, IRR, DPP, optimization, integrated projects.

Language: Russian

Citation: Naumov AA, Naumova AA (2015) TO AN ESTIMATION OF EFFICIENCY OF INNOVATIVE PROJECTS ON THE BASIS OF DETAIL OF FINANCIAL FLOWS. ISJ Theoretical \& Applied Science 06 (26): 85-94.

Soi: http://s-o-i.org/1.1/TAS*06(26)18 Doi: crossef http://dx.doi.org/10.15863/TAS.2015.06.26.18

УДК 330.322.01: 330.322.5:339.944

\section{К ОЦЕНИВАНИЮ ЭФФЕКТИВНОСТИ ИННОВАЦИОННЫХ ПРОЕКТОВ НА ОСНОВЕ МЕТОДА ДЕТАЛИЗАЦИИ ФИНАНСОВЫХ ПОТОКОВ}

Аннотация: Рассмотрены проблемы некоторых методов анализа инвестиций инновациионных проектов на основе приведенного дохода. Предложены схемы оценивания эффективности проектов с учетом интеграции на основе метода детализации финансовых потоков. Исследованы методы оценивания доходности и срока окупаемости проектов. Предложены общие подходы и алгоритмы к оцениванию эффективности интегрированных проектов.

Ключевые слова: Инвестиционные проекты, инновачионные проекты, эффективность, критерии эффективности, NFV, IRR, DPP, оптимизация, интегрированные проекты.

\section{Оценивание инновационных} подходы.

Полный инновационный цикл включает в себя следующие основные этапы: фундаментальные исследования; прикладные исследования; разработка конструкторскотехнологической документации; создание опытного образца; производство опытной партии, маркетинг; запуск массового производства [6][8].

В работе [8] для оценивания эффективности инвестиций инновационного проекта (ИП) предлагается использовать выражение для приведенного дохода (NPV) проекта в виде:

$$
N P V=-I_{n}^{n} \cdot e^{\frac{b \beta \eta}{\gamma}}+\sum_{t=T_{1}+1}^{T} \frac{(1-\tau)\left(R c_{t} S_{t} x+R e n_{t} S_{t} x-S_{f t}\right)}{(1+r)^{t}}
$$

где $\mathrm{I}_{\mathrm{n}}^{\mathrm{n}}-$ объем инвестиций; $\mathrm{e}^{\frac{\mathrm{b} \beta \boldsymbol{\eta}}{\gamma}}-$ мультипликатор для корректировки объема и характера инвестирования инновационного процесса; b - норматив затрат при разработке сложных изделий, непосредственно определяемый на предприятии, $\mathrm{b}>0 ; \quad \beta-$ конструктивная сложность изделия; $\eta$ коэффициент глубины научной проработки 
(теоретические, поисковые, технологические работы), он показывает уровень затрат на финансирование стадий НИОКР; $\gamma$ - показатель интегральной оценки научно-производственного (инновационного) потенциала; Т - время реализации проекта; $\mathrm{t}$ - время, $\mathrm{t} \in\{1,2,3, \ldots, \mathrm{T}\} ; \mathrm{T}_{1}$ - время, затрачиваемое на НИОКР, $\mathrm{T}_{1}<T$; $\tau-$ ставка налога на прибыль; $\mathrm{Rc}_{\mathrm{t}}$ - рентабельность затрат $\left(\mathrm{Rc}_{\mathrm{t}} \mathrm{S}_{\mathrm{t}} \mathrm{x}-\right.$ операционный доход); $\operatorname{Ren}_{\mathrm{t}}-$ «шумпетерианская» рента $\left(\operatorname{Ren}_{\mathrm{t}} \mathrm{S}_{\mathrm{t}} \mathrm{x}-\right.$ рентный доход); $\mathrm{S}_{\mathrm{t}}-$ переменные затраты на одну единицу выпуска нового изделия; $\mathrm{x}$ - предполагаемый объем выпуска новой продукции; $\mathrm{S}_{\mathrm{ft}}-$ часть постоянных затрат, приходящихся на новое изделие; r - уровень планируемой доходности, включающей риски и требования окупаемости (ставка дисконтирования).

Полагая в (1) $N P V=0$, авторы работы [8] получили выражение для оценивания объема инвестиций:

$$
I_{n}=e^{-\frac{b \beta \eta}{\gamma}} \sum_{t=T_{1}+1}^{T_{n}^{n}} \frac{(1-\tau)\left(R c_{t} S_{t} x+R e n_{t} S_{t} x-S_{f t}\right)}{(1+r)^{t}}
$$

Здесь $T_{n}^{n}$ - срок окупаемости проекта.

Сделаем несколько замечаний по поводу формулы (2). Срок окупаемости проекта (точнее - дисконтированный срок окупаемости) $T_{n}^{n}$ определяется как такое наименьшее значение верхней границы Т для индекса суммирования в (1), при котором будет выполняться неравенство $N P V \geq 0$. Отсюда следует, что $T_{n}^{n}$ будет зависеть от объема инвестиций $I_{n}^{n} \cdot e^{\frac{b \beta \eta}{\gamma}}$, т.е. они должны быть известны, для того чтобы оценить $T_{n}^{n}$. Получился своеобразный «замкнутый круг»: для нахождения $I_{n}$ в (2) необходимо знать срок окупаемости $T_{n}^{n}$, а для нахождения последнего необходимо знать объем инвестиций $I_{n}^{n} \cdot e^{\frac{b \beta \eta}{\gamma}}$ из (1).

Нахождение (оценивание) значений параметров в мультипликаторе $e^{\frac{b \beta \eta}{\gamma}}$ представляется задачей достаточно сложной. Для ее решения, в частности, будут малоприемлемы методы регрессионного анализа, поскольку инновационные проекты, как правило, весьма индивидуальны (неповторимы) и, поэтому, использование статистических данных «аналогичных» (похожих) проектов практически невозможно.

Отметим, что использование в качестве критериев эффективности проекта показателей чистого приведенного дохода (NPV), дисконтированного срока окупаемости (здесь $T_{n}^{n}$ ) представляется нерациональным, поскольку эти показатели имеют множество неприятных особенностей. В частности, одна из них связана с выбором ставки дисконтирования r. В работе [8] отмечается, что $\mathrm{r}$ отражает уровень планируемой доходности, включающей риски и требования окупаемости. Как же в таком случае найти оценку фактической доходности проекта? Использование критерия $N P V$ позволяет оценить лишь граничное значение для фактической доходности проектов. Подробнее с проблемами классических показателей эффективности проектов можно ознакомиться по работам [20][22], [29].

Отметим, что в работе [8] основное внимание при исследовании инновационного проекта на эффективность и оценивании инвестиций в проект уделено моменту времени $T_{n}^{n}$ (времени возврата вложений, окупаемости проекта). Именно для этого момента времени и получено выражение (2). Однако, для экономиста-аналитика при анализе инновационных проектов представляется также не менее важной задача нахождения характеристик проекта, относящихся и к другим моментам времени на интервале его жизненного цикла $[1 ; T]$. Исследование поведения таких характеристик в динамике (т.е. оценивание чистого приведенного дохода во времени $N P V(t)$, внутренней нормы доходности $\operatorname{IRR}(t)$ и т.д.) на временном интервале $[1 ; T]$ позволило бы более эффективно управлять проектом.

Мультипликатор $e^{\frac{b \beta \eta}{\gamma}}$ в (1) должен был охарактеризовать поведение инвестиций в зависимости от особенностей инновационного изделия (конструктивной сложности, глубины научной проработки, научно-производственного потенциала). Так, с возрастанием конструктивной сложности ( $\beta$ ) и при неизменных значениях остальных параметров мультипликатора (b, $\eta$ и $\gamma$ ) инвестиции в проект возрастают. Однако из (2) следует, что в этом случае и при фиксированном значении приведенного дохода (постоянства значения суммы в (2)) инвестиции $I_{n}$ будут снижаться. Получилось противоречие с тем, что часто имеет место на практике при реализации инновационных проектов.

\section{Предложения по устранению неточностей} модели (1)-(2).

Что предлагается предпринять для улучшения качества оценок эффективности инновационных проектов? Во-первых, использовать метод детализации финансовых потоков [21]. В этом случае необходимо будет расписать (детализировать, уточнить) прогнозируемые финансовые потоки проекта (инвестиций и доходов) на всем интервале его жизненного цикла. Использование при этом показателей на основе будущего дохода, позволит снять множество проблем, которые возникают при использовании показателей на 
основе чистого приведенного дохода (NPV). Например, проблемы выбора ставки дисконтирования $r$. При формировании финансовых потоков на интервале проведения НИОКР $\left[1 ; T_{1}\right]$ можно будет воспользоваться идеями работы [8] и использовать $\underline{b \beta \eta}$

мультипликаторы вида $e^{\bar{\gamma}}$ или аналогичные им. При этом такие мультипликаторы будут действовать исключительно на входные финансовые потоки проекта (потоки инвестиций, вложений), что на самом деле и отражает особенности этих потоков, как функций от $\beta$ (конструктивной сложности), $\eta$ (глубины научной проработки) и $\gamma$ (научнопроизводственного потенциала). Заметим, что в формуле (2) мультипликатор действует на приведенные выходные (доходные) финансовые потоки проекта, что представляется менее логичным. Поведение выходных потоков проекта в большей степени зависит от характеристик рынка (цен, ставок и пр.).

\section{Оценивание}

эффективности инновационных проектов. Метод детализации финансовых потоков.

Пусть для некоторого инновационного проекта известны входной и выходной финансовые потоки в виде: $F_{i n}(t), \quad t=$ $t_{0}, t_{1}, t_{2}, \ldots, t_{\text {inv }}\left(t_{\text {inv }}<T\right), \quad-\quad$ входной финансовый поток, $F_{\text {out }}(t), \quad t=$ $t_{i n v+1}, t_{i n v+2}, \ldots, T=t_{m}$, - выходной финансовый поток. Заметим, что интервал времени $\left[t_{0} ; t_{i n v}\right]$ включает в себя в качестве подинтервалов моменты времени, относящиеся ко всем стадиям НИОКР (фундаментальное исследование, прикладное исследование, разработка конструкторско-технологической документации, создание опытного образца, производство опытной партии, маркетинг, запуск массового производства). В обозначениях работы [8] эти потоки можно представить в виде $F_{\text {in }}(t)=I_{n t}$, $t=t_{0}, t_{1}, t_{2}, \ldots, t_{\text {inv }}$ (в формулах (1) и (2) $\mathrm{T}_{1}=$ $\left.t_{\text {inv }}\right) \quad$ и $\quad F_{\text {out }}(t)=(1-\tau)\left(R c_{t} S_{t} x+\operatorname{Ren}_{t} S_{t} x-\right.$ $\left.S_{f t}\right), \quad t=t_{\text {inv }+1}, t_{i n v+2}, \ldots, T$. Заметим, что фактически из-за присутствия в потоке $F_{\text {out }}(t)$ его составляющей $S_{f t}$ делает этот поток зависящим от затрат (напомним, что $S_{f t}-$ часть постоянных затрат, приходящихся на новое изделие). Это обстоятельство может быль легко исправлено переносом элементов затрат $\mathrm{S}_{\mathrm{ft}}$ в поток $F_{\text {in }}(t)$, но уже на временном интервале $\left[t_{0} ; T\right]$.

Для простоты записи формул расчета показателей, будем предполагать, что моменты времени $t_{0}, t_{1}, t_{2}, \ldots,\left(t_{m}=T\right)$ - положительные целые числа и все интервалы между соседними отсчетами равны единице времени. Кроме этого полагаем, что все введенные в рассмотрение и используемые ниже ставки согласованы с этой единицей времени. Если обозначить через $r_{i n}$ ставку заимствования инвестируемых финансовых средств, а через $r_{\text {out }}$ - ставку внешнего использования выходного финансового потока, то одна из простейших схем расчета показателя NFV будет выглядеть следующим образом:

$$
\begin{aligned}
& N F V(T)=F_{\text {out }}^{\Sigma}(T)-F_{\text {in }}^{\Sigma}(T) \\
& F_{\text {out }}^{\Sigma}(T)=\sum_{t=t_{\text {inv }+1}}^{t=t_{\text {out }}}(t) \cdot\left(1+r_{\text {out }}\right)^{T-t} \\
& F_{\text {in }}^{\Sigma}(T)=\sum_{t=t_{0}}^{t=t_{\text {inv }}} F_{\text {in }}(t) \cdot\left(1+r_{\text {in }}\right)^{T-t}
\end{aligned}
$$

Заметим, что приведенный доход оценивается на момент времени $T$. Тогда доходность проекта можно оценить по формулам:

$$
I R R=\left\{r \mid \sum_{t} F_{\text {in }}(t) \cdot(1+r)^{T-t}=F_{\text {out }}^{\Sigma}(T)\right\}
$$

или

$$
I R R=\left\{r \mid \sum_{t} F_{\text {in }}(t) \cdot(1+r)^{T-t}=N F V(T)\right\} .
$$

Здесь для простоты записи вместо обозначения суммы в виде $\sum_{t=t_{0}}^{t=t_{i n v}} \ldots$ использовано более компактное обозначение $-\sum_{t} \ldots$. Использование одной из этих формул (4) или (5) зависит от того, хотим ли мы оценить доходность проекта на основании наращенного выходного потока проекта или его общего дохода. Следует заметить, что даже для эффективного бизнеса доходность, оцененная в соответствии с формулой (5), может принимать отрицательные значения. Это объясняется тем обстоятельством, что общий доход $N F V(T)$ может составлять лишь часть общих затрат (инвестиций) в проект $\sum_{t} F_{\text {in }}(t)$.

\section{Детализация финансовых потоков проекта.}

Воспользуемся идеями метода детализации финансовых потоков проекта для оценивания его эффективности. Начнем с детализации входного финансового потока. Заметим, что возможны несколько вариантов реализации процедуры детализации. Рассмотрим один из них.

Будем анализировать последовательно элементы множества $\left\{F_{\text {in }}(t)\right\}, t=t_{0}, t_{1}, t_{2}, \ldots, t_{\text {inv }}$, для возрастающих значений моментов времени. Пусть $F_{\text {in }}\left(t_{0}\right)$ - это заемные средства под ставку $r_{i n, 0}$, которые необходимо будет погашать в виде одинаковых выплат в интервале времени $\left[t_{i n v+1} ; T_{0}\right], t_{i n v+1}<T_{0}$. Хорошо известно, что в этом случае величины кредита и погашений по кредиту связаны между собой следующей формальной записью: 


$$
F_{i n}\left(t_{0}\right) \cdot\left(1+r_{i n, 0}\right)^{t_{i n v+1}-t_{0}+1}=F_{i n, R C, 0} \cdot \frac{1-\left(1+r_{i n, 0}\right)^{-n_{0}}}{r_{i n, 0}} .
$$

Здесь наращенное значение величины кредита (левая часть в равенстве (6)) отражает тот факт, что расчет по нему будет отложен до момента времени $t=t_{i n v+1}$ (точнее - до момента времени близкого к правой границе интервала $\left.\left[t_{i n v+1} ; t_{i n v+2}\right]\right)$, когда начнут поступать доходы от реализации изделия, т.е. по окончании НИОКР; именно в связи с этим обстоятельством в (6) фигурирует показатель степени $\left(t_{i n v+1}-\right.$ $\left.t_{0}+1\right) ; F_{\text {in }, R C, 0}(R C-$ Repayment of a Credit $)-$ выплаты за кредит в размере $F_{i n}\left(t_{0}\right), n_{0}-$

$$
F_{i n, R C, 0}=F_{i n}\left(t_{0}\right) \cdot\left(1+r_{i n, 0}\right)^{t_{i n v+1}-t_{0}+1} \cdot \frac{r_{i n, 0}}{1-\left(1+r_{i n, 0}\right)^{-n_{0}}} .
$$

Таким образом, элемент входного потока $F_{\text {in }}\left(t_{0}\right)$ породил (сгенерировал) через детализацию поток выплат по кредиту: $F_{i n, D F, 0}=$ $\left(F_{i n, R C, 0}, F_{i n, R C, 0}, \ldots, F_{i n, R C, 0}\right)-$ вектор из $n_{0}$ элементов, каждый из элементов вектора привязан к моментам времени $t_{i n v+1}, t_{i n v+2}, \ldots, t_{i n v+n_{0}}=T_{0}$ соответственно.

Переходим к следующему моменту времени $t=t_{1}$ и элементу входного потока $F_{\text {in }}\left(t_{1}\right)$. Предположим, что эти вложения в проект тоже покрываются кредитом со ставкой $r_{i n, 1}$ и количество временных тактов интервала времени $\left[t_{i n v+1} ; T_{0}\right]$, в которые происходят выплаты по кредиту, для целых $t_{i n v+1}$ и $T_{0}$ будет выполняться: $n_{0}=T_{0}-t_{i n v+1}$ (при условии, что кредит выделяется в момент времени $t_{0}$, а погашение его происходит в конце каждого интервала времени $\left[t_{i} ; t_{i+1}\right], i=i n v+1, i n v+$ $2, \ldots$, inv $+n_{0} ;$ где $t_{i n v+n_{0}+1}=T_{0}$.

Из формулы (6) получим значение величин выплат по кредиту:

$$
F_{i n, R C, 1}=F_{i n}\left(t_{1}\right) \cdot\left(1+r_{i n, 1}\right)^{t_{i n v+1}-t_{1}+1} \cdot \frac{r_{i n, 1}}{1-\left(1+r_{i n, 1}\right)^{-n_{1}}}
$$

Допустим, что для момента времени $t=t_{2}$ элемент потока $F_{\text {in }}\left(t_{2}\right)$ будет обеспечен собственными средствами. Поскольку в этом случае имеют дело с собственными средствами, то поток $F_{i n, D F, 2}$ может быть сформирован различными методами (в соответствии с различными схемами): как заемные с некоторой ставкой кредитования $r_{i n, 2}$, в этом случае для представления $F_{\text {in }, D F, 2}$ можно воспользоваться формулами аналогичными вышерассмотренным; как заемные с $r_{i n, 2}=0$ и возвращаемые одномоментно в момент времени $t_{i n v+1}$ (тогда, например, $\quad F_{i n, D F, 2}=\left(F_{i n, R C, 2}=F_{i n}\left(t_{2}\right)\right)$ ). Компонента $F_{i n, R C, 2}$ этого вектора относится ко времени $t=t_{\text {inv }+1}$.

Процедура детализации элементов входного потока продолжает свою работу до момента времени $t=t_{\text {inv }}$ (в результате получится вектор $\left.F_{i n, D F, i n v}\right)$.

После этого следует свести (объединить, слить) все векторы $F_{i n, D F, i}, i=0,1,2, \ldots, i n v$, определенные на интервале $\left[t_{i n v+1} ; T\right]$, в один общий вектор с учетом привязки компонент этих временным интервалом его погашения $\left[t_{\text {inv+1 }} ; T_{1}\right]$, причем для целых $t_{i n v+1}$ и $T_{1}$ количество выплат по второму кредиту равно $n_{1}=T_{1}-t_{i n v+1}$. Тогда элемент входного потока $F_{\text {in }}\left(t_{1}\right)$ в результате его детализации приведет к образованию вектора выплат вида: $F_{i n, D F, 1}=$ $\left(F_{i n, R C, 1}, F_{i n, R C, 1}, \ldots F_{i n, R C, 1}\right)$, элементы которого относятся к моментам времени $t_{i n v+1}, t_{i n v+2}, \ldots, t_{i n v+n_{1}}=T_{1}$ соответственно. $\mathrm{B}$ этом векторе векторов к моментам времени и, суммируя значения компонент этих векторов, относящихся к одним моментам времени. Если для некоторого момента времени $t_{i n v+j}$ из интервала $\left[t_{i n v+1} ; T\right]$ значение компоненты вектора $F_{i n, D F, i}, i \in$ $\{0,1,2, \ldots$, inv $\}$ не определено, то оно полагается равным нулю. Понятно, что в общем случае число компонент в новом свернутом векторе может быть больше общего числа временных отсчетов $\left(T-t_{\text {inv+1 }}\right)=\left(t_{m}-t_{\text {inv }+1}\right)$. Это означает, что элементы входного потока, относящиеся к моментам времени следующими за моментом $t_{m}$, будут погашаться за пределами горизонта рассмотрения потоков проекта, т.е. за границами временного интервала $\left[t_{0} ; T\right]$. Подобные ситуации требуют проведения дополнительных исследований.

Итак, в результате свертки векторов $F_{i n, D F, i}, i=0,1,2, \ldots, i n v$, получим новый вектор $F_{\text {in, } D F}^{\Sigma}$. Условно эту свертку векторов обозначим следующим образом: $F_{i n, D F}^{\Sigma}=\oplus_{i=0}^{i n v} F_{i n, D F, i}$. Для определенности положим, что компоненты вектора $F_{i n, D F}^{\Sigma}$ относятся к моментам времени $t=$ 
$t_{i n v+1}, t_{i n v+2}, \ldots, t_{D F}=T_{D F}$ и, как было отмечено выше, в общем случае может выполняться неравенство $T_{D F} \geq T$.

Перейдем к детализации элементов выходного потока $F_{\text {out }}(t), \quad t=$ $t_{i n v+1}, t_{i n v+2}, \ldots, t_{m}$. Основная идея процедуры детализации выходного потока состоит в том, чтобы распределить элементы этого потока в два. Один поток будет показывать, как погашаются элементы потока $F_{\text {in,DF }}^{\Sigma}$ (обозначим его как $F_{o u t, I P}^{\Sigma}$, где IP - Internal Projects, внутренние проекты). Второй - поток, который может быть выведен из данного проекта и использован в других проектах (обозначим его как $F_{\text {out,EP}}^{\Sigma}$, где EP - External Projects, внешние проекты). Следует иметь в виду, что наряду с непосредственно элементами этих двух потоков $\left(F_{\text {out }, I P}^{\Sigma}\right.$ и $\left.F_{\text {out }, E P}^{\Sigma}\right)$ в результате применения процедуры детализации будут получены два соответствующих им вектора параметров $-\pi_{\text {out }, I P}^{\Sigma}$ и $\pi_{o u t, E P}^{\Sigma}$. Эти параметры представляют собой ставки, по которым элементы $F_{\text {out,IP }}^{\Sigma}$ ожидают их использования в качестве погашений потока $F_{i n, D F}^{\Sigma}$ (это параметры $\pi_{\text {out,IP }}^{\Sigma}$, а элементы $F_{\text {out,EP }}^{\Sigma}-$ используются во внешних проектах (за это отвечают параметры

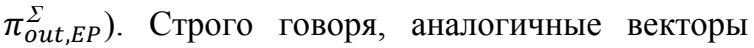
параметров сопровождают и компоненты вектора $F_{i n, D F}^{\Sigma}$ (и векторов $\left.F_{i n, D F, i}, i=0,1,2, \ldots, i n v\right)$. Для этих векторов параметры характеризуют элементы входного потока: ставки заимствования - $r_{i n, i}, i=0,1,2, \ldots, i n v$; количество временных тактов погашения кредитов $n_{i}, i=0,1,2, \ldots, i n v$. Как и для элементов входного потока, в данном случае можно записать равенство: $F_{\text {out }, I P}^{\Sigma}=$ $\oplus_{i=\text { inv }+1}^{m} F_{\text {out }, I P, i} \quad$ и $\quad F_{\text {out }}=F_{\text {out }, I P}^{\Sigma} \oplus F_{\text {out }, E P}^{\Sigma}$. Здесь $F_{\text {out }}=\left(F_{\text {out }}\left(t_{\text {inv }+1}\right), F_{\text {out }}\left(t_{\text {inv }+2}\right), \ldots, F_{\text {out }}\left(t_{m}\right)\right)$.

Очевидно, что элементы вектора $F_{o u t, I P}^{\Sigma}$ должны полностью компенсировать (быть равны) компоненты (компонентам) вектора $F_{i n, D F}^{\Sigma}$ для всех моментов времени $t=t_{i n v+1}, t_{i n v+2}, \ldots, t_{m}$. Формально это можно записать таким образом: $F_{\text {out }, I P}^{\Sigma}(t)=F_{\text {in,DF }}^{\Sigma}(t) \quad$ для $\quad$ всех $\quad t \in$ $\left\{t_{i n v+1}, t_{i n v+2}, \ldots, t_{m}\right\}$. Если это условие не выполняется, то необходимо повторить процедуру детализации сначала (поменяв источники кредитования, условия кредитования и т.д.) или, сделать вывод о том, что проект не является эффективным. Последнее означает, что его выходной поток (поток доходов) не может компенсировать входной поток (поток вложений).

Если равенства $F_{\text {out }, I P}^{\Sigma}(t)=F_{i n, D F}^{\Sigma}(t)$ для всех $t \in\left\{t_{i n v+1}, t_{i n v+2}, \ldots, t_{m}\right\}$ выполнились, то можно перейти к оцениванию показателей эффективности проекта. В частности, можно оценить доход проекта $N F V_{D F}$ в соответствии с формулой:

$$
N F V_{D F}=\sum_{t=t_{\text {inv }+1}}^{T} F_{\text {out }, E P}^{\Sigma}(t) \cdot\left(1+r_{\text {out }, E P}(t)\right)^{T-t} .
$$

Здесь $F_{\text {out,EP }}^{\Sigma}(t)$ - элемент вектора $F_{o u t, E P}^{\Sigma}$, относящийся к моменту времени $t, r_{\text {out,EP }}(t)-$ ставка внешнего использования (во внешних проектах) средств в размере $F_{o u t, E P}^{\Sigma}(t)$. Заметим, что в общем случае таких ставок может быть несколько, они соответствуют доходностям проектов, в которые вкладываются средства $F_{\text {out }, E P}^{\Sigma}(t)$.

Отметим положительные моменты, связанные с показателем $N F V_{D F}$, рассчитанным в соответствии с формулой (9):

1. Все ставки, используемые в расчетах этого показателя, имеют ясный смысл и выбираются достаточно однозначно (это либо банковские ставки для кредитов, либо ставки по депозитам, либо ставки внешних проектов, эффективность которых оценена и т.д.). Если какие-либо из этих ставок точно не известны, то в этом случае следует наряду с самим значением показателя $N F V_{D F}$ оценить для него и риск $\Re_{N F V_{D F}}$ (так, как это предлагается делать, например, в [21]). А затем, при анализе проекта на эффективность и выборе лучшего проекта из множества альтернативных проектов оперировать парой доход-риск $\left\langle N F V_{D F}, \Re_{N F V_{D F}}\right\rangle$.

2. Значение показателя тоже интерпретируется достаточно просто: это доход от проекта, который будет получен на момент времени $t=T$. В связи с этим обстоятельством показатель можно было бы обозначить еще и таким образом - $N F V_{D F}(T)$, введя в его обозначение время окончания проекта или время, на которое проводится анализ проекта на эффективность.

3. Фактически элементы $F_{\text {out,EP }}^{\Sigma}(t)$ в формуле для показателя (9) равны разностям между компонентами детализированных векторов выходного и входного потоков.

Опираясь на выражение (9) для оценивания дохода $N F V_{D F}$, можно предложить расчетные схемы для оценивания доходности проекта, срока его окупаемости и т.д.

Так, например, для оценивания доходности проекта можно воспользоваться формулами: 
$I R R_{D F, i n, N F V+D F}=\left\{r \mid \begin{array}{l}\sum_{t} F_{i n}(t) \cdot(1+r)^{T-t}= \\ =N F V_{D F}+\sum_{t} F_{i n, D F}^{\Sigma}(t)\end{array}\right\}$,

$I R R_{D F, \text { in }}=\left\{r \mid \sum_{t} F_{\text {in }}(t) \cdot(1+r)^{T-t}=N F V_{D F}\right\}$,

$I R R_{D F, D F}=\left\{r \mid \sum_{t} F_{i n, D F}^{\Sigma}(t) \cdot(1+r)^{T-t}=N F V_{D F}\right\}$.

Эти три формулы отличаются тем, какой из потоков (погашения кредитов и входной поток инвестиций в проект или каждый из них в отдельности) положен в основу оценивания доходности проекта. Во всех случаях дается ответ на вопрос, каково влияние потоков $\left(\left(F_{i n}(t)+\right.\right.$ $\left.F_{i n, D F}^{\Sigma}(t)\right), F_{i n}(t)$ или $\left.F_{i n, D F}^{\Sigma}(t)\right)$ на общий доход проекта $\left(N F V_{D F}\right)$.

Оценивание срока окупаемости проекта. Формально для оценки срока окупаемости можно записать определение в виде:

$$
P P=\left\{\begin{array}{c}
\min t^{*} \in\left\{t_{i n v+1}, t_{i n v+2}, \ldots, t_{m}=T\right\} \\
\vdots \quad N F V_{D F}(t) \geq F_{i n}^{\Sigma}(t), \\
\forall_{t} t \in\left\{t^{*}, t^{*}+1, t^{*}+2, \ldots, t_{m}=T\right\}
\end{array}\right\}
$$

Здесь, как и выше, $\quad N F V_{D F}(t)=$ $\sum_{\tau=t_{\text {inv }+1}}^{\tau=t} F_{\text {out }, E P}^{\Sigma}(\tau) \cdot\left(1+r_{\text {out }, E P}(\tau)\right)^{T-\tau}$ - доход от проекта на момент времени $t \in$ $\left\{t_{i n v+1}, t_{i n v+2}, \ldots, t_{m}=T\right\}, \quad$ a $\quad F_{i n}^{\Sigma}(t)=$ $\sum_{\tau=t_{\text {inv }+1}^{\tau}}^{\tau=t} F_{\text {in }}(\tau) \cdot\left(1+r_{\text {in,alt }}\right)^{T-\tau}, \quad$ где $\quad r_{\text {in,alt }} \quad-$ альтернативная ставка использования элементов входного потока (инвестиций), возможно, что будет положено $r_{\text {in alt }}=0$.

Можно определить срок окупаемости и как такой момент времени, на который будет получен доход, превосходящий не только суммарные инвестиции в проект (как это сделано в (11)), но и все выплаты за заемные средства (с учетом потока $\left.\left(F_{i n}(t)+F_{i n, D F}^{\Sigma}(t)\right)\right)$. В этом случае расчетная формула для срока окупаемости проекта будет иметь вид:

$$
P P=\left\{\begin{array}{c}
\min t^{*} \in\left\{t_{i n v+1}, t_{i n v+2}, \ldots, t_{m}=T\right\} \vdots \\
\vdots \quad N F V_{D F}(t) \geq F_{i n}^{\Sigma}(t)+F_{i n, D F}^{\Sigma, \Sigma}(t), \\
\forall_{t} t \in\left\{t^{*}, t^{*}+1, t^{*}+2, \ldots, t_{m}=T\right\}
\end{array}\right\} .
$$

Здесь $\cdot\left(1+r_{\text {out }, E P}(\tau)\right)^{T-\tau}-$ доход от проекта на момент времени $t \in\left\{t_{i n v+1}, t_{i n v+2}, \ldots, t_{m}=T\right\}$, $F_{\text {in }}^{\Sigma}(t)=\sum_{\tau=t_{\text {inv+1 }}}^{\tau=t} F_{\text {in }}(\tau) \cdot\left(1+r_{\text {in,alt }}\right)^{T-\tau}$, $F_{i n, D F}^{\sum, \Sigma}(t)=\sum_{\tau=t_{i n v+1}}^{\tau=t} F_{i n, D F}^{\Sigma}(\tau) \cdot\left(1+r_{\text {in,alt }}\right)^{T-\tau}$, где $r_{\text {in,alt }}$ - альтернативная ставка использования элементов входного потока (инвестиций) и средств, пошедших на погашение кредитов. Заметим, что ставки $r_{\text {in,alt }}$ в двух последних формулах могут быть различными (несовпадающими). Кроме этого, можно предложить и другие подходы к оцениванию срока окупаемости проекта (см., например, [21],[22]).

Оценивание доходов и доходностей для интегрированных инновационных проектов.

Интеграция проектов при реализации НИОКР и последующей его эксплуатации явление обыденное. В этом случае различные этапы НИОКР будут возложены на различных исполнителей. И, таким образом, финансовая нагрузка по проекту (в частности, по его инвестированию) распределяется между участниками интеграции. Конечно, такая интеграция подразумевает, что каждый из исполнителей обладает наилучшим потенциалом для исполнения отведенного ему этапа НИОКР (своеобразного подпроекта общего проекта). Эти этапы участников интеграции могут налагаться (накладываться, пересекаться) во времени, но все они укладываются во временной интервал $\left[t_{0} ; t_{i n v}\right]$. Если число участников интеграции $N_{\text {int }}$, то для каждого из участников можно будет расписать его входной финансовый поток, а все вместе они будут образовывать множество $F_{\text {in, } j}(t), j=1,2,3, \ldots, N_{\text {int }} ; t=t_{0}, t_{1}, t_{2}, \ldots, t_{\text {inv }}$.

При этом, без умаления общности, предполагаем, что выходной финансовый поток интегрированного проекта остается один $F_{\text {out }}(t), t=t_{i n v+1}, t_{i n v+2}, \ldots, t_{m} . \quad$ Тогда, при использовании метода детализации потоков каждый из входных потоков участников интеграционного процесса породит поток вида $F_{\text {in }, D F, i, j}, i=0,1,2, \ldots$, inv,$\quad$ где $j \in\left\{1,2, \ldots, N_{\text {int }}\right\}$. Размерность задачи (модели) в этом случае увеличивается, но в работе алгоритма метода детализации потоков принципиальных изменений не произойдет.

Например, можно предложить следующий алгоритм для оценивания показателей эффективности интегрированного проекта. Распишем его по шагам.

1 шаг. Для интегрированной структуры из проектов (составленной из проектов каждого из участников интеграции) находим доход $\left(N F V_{D F, I}\right)$ и доходность $\left(I R R_{D F, I}\right)$ на основании метода детализации потоков рассмотренного выше. Если значения этих показателей устраивают всех потенциальных участников интеграционного процесса, то переходим на 2-ой шаг, иначе - на 5ый шаг (с результатом - «Не реализовывать интеграционный проект»).

2 шаг. Используя доходность интегрированного проекта $\left(I R R_{D F, I}\right)$ и входные потоки каждого из частных проектов $\left(F_{i n, j}(t), j=\right.$ $\left.1,2,3, \ldots, N_{\text {int }}\right)$, находим их доходы по формуле: 
$N F V_{D F, j}=\sum_{t} F_{i n, j}(t) \cdot\left(1+I R R_{D F, I}\right)^{T-t}$, $j=1,2,3, \ldots, N_{\text {int }}$.

3 шаг. Доходности каждого из частных проектов находим по одной из формул (10).

4 шаг. Если каждого из участников процесса устраивают значения показателей (дохода и доходности) для их частных проектов, то принимается решение о заключении формальных договоров, составлении проектной документации и реализации интеграционного проекта. Иначе переходим на 5-ый шаг с решением: «В интеграционном проекте не участвовать»).

5 шаг. Конец работы алгоритма анализа интегрированного проекта на эффективность и принятия решения о реализации (или не реализации) интеграционного процесса.

Пример. Пусть заданы финансовые потоки проекта (см. Табл.1).

Потоки проекта.

Таблица 1

\begin{tabular}{|c|c|c|c|c|c|}
\hline$t$ & $t_{0}$ & $t_{1}$ & $t_{2}$ & $t_{3}$ & $t_{4}$ \\
\hline$F_{\text {in }}(t)$ & 100 & 50 & 50 & 0 & 0 \\
\hline$F_{\text {out }}(t)$ & 0 & 70 & 90 & 100 & 100 \\
\hline
\end{tabular}

Заметим, что в этом примере $t_{0}=t_{\text {inv }}$ и для моментов времени $t_{1}$ и $t_{2}$ учтены затраты, связанные с сопровождением проекта (расширением производственной базы, увеличением объемов выпуска продукции и т.д.).

Пусть для момента времени $t=t_{0}$ известны $r_{i n, 0}=0.2$ и $n_{0}=2$. Находим $F_{i n, R C, 0}=F_{i n}\left(t_{0}\right)$. $\frac{r_{i n, 0}}{1-\left(1+r_{i n, 0}\right)^{-n_{0}}}=100 \cdot \frac{0.2}{1-(1+0.2)^{-2}}=65.4545 \quad$ (ден. ед.). Составляем вектор детализации выплат по проекту для $t=t_{0}$ получим $\quad F_{i n, D F, 0}=$ $\left(0, F_{i n, R C, 0}, F_{\text {in }, R C, 0}\right)=(0,65.4545,65.4545)$.

Для момента времени $t=t_{1}$ при $r_{i n, 1}=0.1$, $n_{1}=3$ находим $F_{i n, R C, 1}=F_{i n}\left(t_{1}\right) \cdot \frac{r_{i n, 1}}{1-\left(1+r_{i n, 1}\right)^{-n_{1}}}=$
$50 \cdot \frac{0.1}{1-(1+0.1)^{-3}}=20.1057 \quad$ (ден. $\quad$ ед.). Тогда $F_{i n, D F, 1}=\left(0, F_{i n, R C, 1}, F_{i n, R C, 1}, F_{i n, R C, 1}\right)=$ $(0,20.1057,20.1057,20.1057)$.

Наконец, для $\mathrm{t}=\mathrm{t}_{2}$ при $\mathrm{r}_{\mathrm{in}, 2}=0.2, \mathrm{n}_{2}=2$ находим $\quad \mathrm{F}_{\mathrm{in}, \mathrm{RC}, 2}=\mathrm{F}_{\mathrm{in}}\left(\mathrm{t}_{2}\right) \cdot \frac{\mathrm{r}_{\mathrm{in}, 2}}{1-\left(1+\mathrm{r}_{\mathrm{in}, 2}\right)^{-\mathrm{n}_{2}}}=50$. $\frac{0.2}{1-(1+0.2)^{-2}}=32.7273$ (ден. ед.). Тогда $\mathrm{F}_{\mathrm{in,DF}, 2}=$ $\left(0, \mathrm{~F}_{\mathrm{in}, \mathrm{RC}, 2}, \mathrm{~F}_{\mathrm{in}, \mathrm{RC}, 2}\right)=(0,32.7273,32.7273)$.

Находим свертку $\quad \mathrm{F}_{\mathrm{in}, \mathrm{DF}}^{\Sigma}=\oplus_{\mathrm{i}=0}^{\mathrm{m}} \mathrm{F}_{\mathrm{in}, \mathrm{DF}, \mathrm{i}}=$ $(0,65.4545,85.5602,52.8330,52.8330)$. Покажем этот вектор и вектор выходного потока в новой таблице (см. Табл. 2).

Результаты детализации выходного потока проекта сведем в Табл. 3 .

\section{Детализированные входной и исходный выходной потоки проекта.}

Таблица 2

\begin{tabular}{|c|c|c|c|c|c|}
\hline $\mathrm{t}$ & $\mathrm{t}_{0}$ & $\mathrm{t}_{1}$ & $\mathrm{t}_{2}$ & $\mathrm{t}_{3}$ & $\mathrm{t}_{4}$ \\
\hline $\mathrm{F}_{\text {in,DF }}^{\Sigma}$ & 0 & 65.454 & 85.560 & 52.833 & 52.833 \\
\hline $\mathrm{F}_{\text {out }}(\mathrm{t})$ & 0 & 70 & 90 & 100 & 100 \\
\hline
\end{tabular}

Таблица 3

\section{Детализированные входной и выходной потоки проекта.}

\begin{tabular}{|c|c|c|c|c|c|}
\hline $\mathrm{t}$ & $\mathrm{t}_{0}$ & $\mathrm{t}_{1}$ & $\mathrm{t}_{2}$ & $\mathrm{t}_{3}$ & $\mathrm{t}_{4}$ \\
\hline $\mathrm{F}_{\text {in,DF }}^{\Sigma}$ & 0 & 65.454 & 85.560 & 52.833 & 52.833 \\
\hline $\mathrm{F}_{\text {out,IP }}^{\Sigma}$ & 0 & 65.454 & 85.560 & 52.833 & 52.833 \\
\hline $\mathrm{F}_{\text {out,EP }}^{\Sigma}$ & 0 & 4.545 & 4.439 & 47.167 & 47.167 \\
\hline
\end{tabular}

Тогда для $\mathrm{r}_{\mathrm{out}, \mathrm{EP}}(\mathrm{t})=0.5, \quad \mathrm{t} \in\left\{\mathrm{t}_{0}, \mathrm{t}_{1}, \ldots, \mathrm{t}_{4}\right\}$, находим доход проекта:

$$
\mathrm{NFV}_{\mathrm{DF}}=\sum_{\mathrm{t}} \mathrm{F}_{\text {out,EP }}^{\Sigma}(\mathrm{t}) \cdot\left(1+\mathrm{r}_{\text {out }, \mathrm{EP}}(\mathrm{t})\right)^{\mathrm{T}-\mathrm{t}}=
$$

143.2481 (ден. ед.).

Используя формулу (10) и альтернативные формулы, находим доходность проекта: 


$$
\begin{gathered}
\operatorname{IRR}_{\mathrm{DF}, \mathrm{in}, \mathrm{NFV}+\mathrm{DF}}=\left\{\mathrm{r} \mid \sum_{\mathrm{t}} \mathrm{F}_{\mathrm{in}}(\mathrm{t}) \cdot(1+\mathrm{r})^{\mathrm{T}-\mathrm{t}}=\mathrm{NFV}_{\mathrm{DF}}+\sum_{\mathrm{t}} \mathrm{F}_{\mathrm{in}, \mathrm{DF}}^{\Sigma}(\mathrm{t})\right\}=0.2321 \quad \text { (или 23.21\% ), } \\
\operatorname{IRR}_{\mathrm{DF}, \text { in }}=\left\{\mathrm{r} \mid \sum_{\mathrm{t}} \mathrm{F}_{\mathrm{in}}(\mathrm{t}) \cdot(1+\mathrm{r})^{\mathrm{T}-\mathrm{t}}=\mathrm{NFV}_{\mathrm{DF}}\right\}=-0.098635
\end{gathered}
$$

И

$$
\mathrm{IRR}_{\mathrm{DF}, \mathrm{DF}}=\left\{\mathrm{r} \mid \sum_{\mathrm{t}} \mathrm{F}_{\mathrm{in}, \mathrm{DF}}^{\Sigma}(\mathrm{t}) \cdot(1+\mathrm{r})^{\mathrm{T}-\mathrm{t}}=\mathrm{NFV}_{\mathrm{DF}}\right\}=-0.3422
$$

Отрицательные значения этого показателя (для $\mathrm{IRR}_{\mathrm{DF}, \text { in }}$ и $\mathrm{IRR}_{\mathrm{DF}, \mathrm{DF}}$ ) говорят о том, что доход проекта $\mathrm{NFV}_{\mathrm{DF}}=143.2481 \quad$ (ден. ед.) не превышает вложений в этот проект в качестве входного потока и потока расчета по кредитам потоки $\mathrm{F}_{\text {in }}$ и $\mathrm{F}_{\mathrm{in}, \mathrm{DF}}^{\Sigma}$ (см. Табл. 1 и Табл. 3). Заметим, что $\mathrm{NFV}_{\mathrm{DF}}+\sum_{\mathrm{t}} \mathrm{F}_{\mathrm{in,DF}}^{\Sigma}(\mathrm{t})=399.9288$ (ден. ед.).

И наконец, в соответствии с формальной записью (11) оцениваем значение срока окупаемости данного проекта:

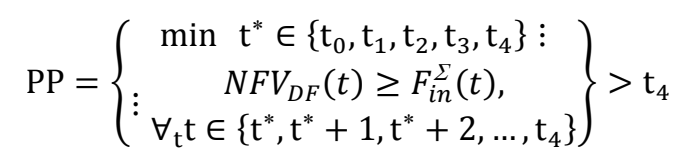

Сделаем некоторые выводы по рассмотренному примеру.

1. Доход и доходность по данному проекту не велики, поскольку в проекте использованы только заемные средства.

2. Несмотря на то, что внешний проект, куда направляются заработанные в данном проекте средства, имеет высокую доходность $\left(\mathrm{r}_{\text {out,EP }}(\mathrm{t})=0.5\right)$, общий доход не столь велик, поскольку в моменты времени $t_{1}$ и $t_{2}$ в него выводятся из основного проекта небольшие средства (в количестве 4.545 ден. ед. и 4.439 ден. ед. соответственно).

3. Задача оптимизации проекта может состоять в выборе таких его параметров (ставок, сроков и т.д.), которые привели бы к увеличению значений его показателей, например, $\mathrm{NFV}_{\mathrm{DF}}$ и $\mathrm{IRR}_{\mathrm{DF}, \mathrm{in}, \mathrm{NFV}+\mathrm{DF}}$.

4. Интересным с точки зрения повышения эффективности данного проекта может быть, например, такой вариант. Поскольку доходность внешнего проекта $\left(\mathrm{r}_{\text {out,EP }}(\mathrm{t})=\right.$ $0.5)$ составляет величину большую, чем ставки заемного процента $\left(\mathrm{r}_{\mathrm{in}, 0}=0.2, \mathrm{r}_{\mathrm{in}, 1}=\right.$ 0.1 и $\mathrm{r}_{\mathrm{in}, 2}=0.2$ для трех заимствований соответственно), то можно предложить погашать кредиты новыми кредитами. Еще один прием повысить эффективность проекта - увеличить длительности периодов времени расчета по кредитам. Оба эти варианта действий приведут к тому, что большие объемы средств могут быть переведены во внешний проект. Сделаем немаловажную оговорку: необходимо, чтобы этот внешний проект был в состоянии освоить эти увеличившиеся объемы перечислений. На этапе проектирования и исходного и внешнего проектов это сделать проще, чем в то время, когда внешний проект уже реализуется.

5. Поскольку многие переменные, которые можно подбирать (изменять) в проекте, имеют дискретный характер, то задача оптимизации проекта представляет собой переборную задачу (задачу дискретного программирования, задачу выбора лучшего варианта из конечного множества альтернатив).

6. Очень интересными представляются следующие задачи. Как учесть привязку элементов потока $\mathrm{F}_{\mathrm{in,DF}}^{\Sigma}$ ко времени? Более точно - как учесть в каком объеме и в какие моменты времени генерируются элементы потока $\mathrm{F}_{\mathrm{in}, \mathrm{DF}}^{\Sigma}$ ? Заметим, что в выражении $\left(\mathrm{NFV}_{\mathrm{DF}}+\sum_{\mathrm{t}} \mathrm{F}_{\mathrm{in,DF}}^{\Sigma}(\mathrm{t})\right), \quad$ которое было использовано для оценивания доходности проекта, элементы этого потока были просуммированы без учета временного фактора.

Перечислим еще некоторые интересные с теоретической и практической точек зрения задачи анализа ИП: задача оптимизации структуры капитала ИП [14], [15], [22], [23], [24], задача оценивания эффективности интегрированных проектов [16], [17], [19], [21], [24], [25], [29], задача выбора критериев эффективности ИП [12], [13], [18], [25], [26], [27].

\section{Выводы.}

1. Рассмотрены проблемы предлагаемых методов анализа инвестиций ИП на основе приведенного дохода (1)-(2). Предложены схемы оценивания эффективности проектов с учетом интеграции на основе метода детализации финансовых потоков.

2. Исследованы методы оценивания доходности и срока окупаемости проектов.

3. Предложены общие подходы и алгоритмы к оцениванию эффективности интегрированных проектов. 


\section{References:}

1. Vilenskiy PL, Livshits VN, Smolyak SA (2001) Otsenka effektivnosti investitsionnyih proektov: teoriya i praktika: uchebno-praktich. posobie. Moscow. Delo, pp. 451.

2. Lipsits IV, Kossov VV (2003) Ekonomicheskiy analiz realnyih investitsiy. Moscow, Ekonomist. pp. 341.

3. Rimer MI, Kasatov AD, Matienko NN (2008) Ekonomicheskaya otsenka investitsiy. $\mathrm{SPb}$. Piter, pp. 480.

4. Kalmyikova TS (2009) Investitsionnyiy analiz: ucheb. posobie. Moscow. INFRA-M, pp. 204.

5. Kovalev VV (2005) Kurs finansovyih vyichisleniy: ucheb. posobie. Moscow. Finansyi i statistika, pp. 354.

6. Pleshhinskij AS (2004) Optimizacija mezhfirmennyh vzaimodejstvij i vnutrifirmennyh upravlencheskih reshenij. Moscow. Nauka, pp. 252.

7. Titov VV (2007) Optimizacija upravlenija promyshlennoj korporaciej: voprosy metodologii i modelirovanija. Novosibirsk. IJeOPP SO RAN, pp. 256.

8. Rastova JI, Mezhov SI (2014) Metodicheskie osnovy prognoznoj ocenki ob \#ema investirovanija $\mathrm{v}$ innovacionnye proekty. Vestnik SPbGJeU, Ser. Jekonomika, Vyp. 2(69), pp. 78-83.

9. Titov VV, Mezhov SI (2013) Model' formirovanija operacionno-innovacionnoj programmy promyshlennogo predprijatija // Sovershenstvovanie institucional'nyh mehanizmov upravlenija $\mathrm{v}$ promyshlennyh korporacijah/ Pod. red. V.V.Titova, V.D. Markovoj. Novosibirsk. IJeOPP SO RAN, pp. 232-239.

10. Mezhov SI, Nezhinskij OM (2013) Investicionnye strategii i ocenka ih jeffektivnosti// Problemy teorii i praktiki upravlenija. No. 5, pp. 101-106.

11. Mezhov SI (2012) Jekonomika innovacionnoj korporacii: teorija i problemy jeffektivnosti. Barnaul. Izd-vo AAJeP, pp. 216.

12. Naumov AA (2013) O tochnosti otsenok sroka okupaemosti investitsionnyih proektov. Theoretical\&Applied Science, Materials of the ISPC «Results \&Perspectives», 30.09.2013, Florence, Italy, No. 9 (5), pp. 95-99. doi: http://dx.doi.org/10.15863/TAS.2013.09.5.10

13. Naumov AA (2013) K voprosu o tochnosti otsenki diskontirovannogo sroka okupaemosti investitsionnogo proekta. Finansovaya analitika: problemyi i resheniya, No. 44 (182), pp. 25-28.
14. Naumov AA (2013) K analiticheskim resheniyam nekotoryih ekonomikomatematicheskih zadach. Theoretical\&Applied Science, Materials of the ISPC «Results \&Perspectives», 30.09.2013, Florence, Italy, No. 9 (5), pp. 99-104. doi: http://dx.doi.org/10.15863/TAS.2013.09.5.11

15. Naumov AA (2013) K voprosu ob uproschenii dvuh zadach optimizatsii investitsiy. Finansovaya analitika: problemyi i resheniya, No. 46 (184), pp. 26-30.

16. Naumov AA (2013) K modelyam sovmestnogo upravleniya proektami. Theoretical\&Applied Science, Materials of the ISPC «Theory and Practice», 30.08.2013, Munich, Germany, No. 8 (4), pp. 90-93. doi: http://dx.doi.org/10.15863/TAS.2013.08.4.11

17. Naumov AA, Kryukov SV (2013) K problemam odnoy modeli upravleniya sovmestnyimi investitsionnyimi proektami. Finansovaya analitika: problemyi i resheniya, No. 40 (178), pp. 53-56.

18. Naumov AA (2013) Ispolzovanie metoda detalizatsii finansovyih potokov $\mathrm{v}$ zadachah otsenivaniya effektivnosti proektov. Finansovaya analitika: problemyi i resheniya, No. 48 (186), pp. 35-41.

19. Naumov AA (2012) K zadache otsenivaniya chastnyih effektov integrirovannyih biznesprotsessov. Finansovaya analitika: Problemyi i resheniya, No. 46 (136), pp. 41-48.

20. Naumov AA (2012) Teoreticheskie i prikladnyie voprosyi modelirovaniya biznesprotsessov. Modeli, algoritmyi, programmyi. LAP LAMBERT Academic Publishing, pp. 464.

21. Naumov AA (2013) Metodyi analiza i sinteza investitsionnyih proektov. Effektivnost, riski, upravlenie. LAP LAMBERT Academic Publishing, pp. 356.

22. (2015) Spisok trudov. Available: https://sites.google.com/site/anatolynaumov201 1/home/spisok-trudov-list-of-papers (Accessed 27.06.15).

23. Naumov AA, Naumova AA (2014) O nekorrektnosti odnoy modeli optimizatsii strukturyi kapitala. ISJ Theoretical\&Applied Science, Materials of the ISPC «European Innovation», 30.09.2014, Martigues, France, No. 9 (17), pp. 170-173. doi: http://dx.doi.org/10.15863/TAS.2014.09.17.29

24. Naumov AA (2013) Optimizatsiya strukturyi zaimstvovaniy i vlozheniy dohodov investitsionnogo proekta. Theoretical \& Applied Science, Materials of the ISPC 
Impact Factor ISRA (India) $\quad=\mathbf{1 . 3 4 4}$

Impact Factor ISI (Dubai, UAE) $=\mathbf{0 . 8 2 9}$

based on International Citation Report (ICR)

Impact Factor GIF (Australia) $\quad \mathbf{0} \mathbf{0 . 3 5 6}$

«Advances in techniques\&technologies», 30.10.2013, Milan, Italy, No. 10(6), pp. 133136. doi: http://dx.doi.org/10.15863/TAS.2013.10.6.20

25. Naumov AA (2014) Otsenivanie effektivnosti integrirovannyih proektov. Finansovaya analitika: problemyi i resheniya, No. 8 (194), pp. 36-43

26. Naumov AA (2014) Analiz kriteriev effektivnosti investitsionnyih proektov. ISJ Theoretical\&Applied Science, Materials of the ISPC «Modern mathematics in science», 30.06.2014, Caracas, Venezuela, No. 6 (14), pp. 92-94. doi: http://dx.doi.org/10.15863/TAS.2014.06.14.19

27. Naumov AA, Bazhenov RI (2014) O problemah klassicheskih pokazateley effektivnosti $\begin{array}{lr}\text { Impact Factor JIF } & =\mathbf{1 . 5 0 0} \\ \text { Impact Factor SIS }(\text { USA) } & =\mathbf{0 . 9 1 2} \\ \text { Impact Factor PИНЦ (Russia) } & =\mathbf{0 . 1 7 9} \\ \text { Impact Factor ESJI }(\text { KZ }) & =\mathbf{1 . 0 4 2}\end{array}$

investitsionnyih proektov. Sovremennyie nauchnyie issledovaniya i innovatsii, No. 11 Available:

http://web.snauka.ru/issues/2014/11/40825 (Accessed: 20.11.2014).

28. Naumov AA, Bazhenov RI (2014) O neustoychivosti metoda normalizatsii kriteriev. Sovremennyie nauchnyie issledovaniya i innovatsii, No. $11 . \quad$ Available: http://web.snauka.ru/issues/2014/11/40408 (Accessed: 10.11.2014).

29. Naumov AA (2014) Upravlenie portfel'nymi investicijami. Jeffektivnost', riski, optimizacija. LAP LAMBERT Academic Publishing, Saarbrucken, pp. 236. 